\title{
Production of erythriod cells from human embryonic stem cells by fetal liver cell extract treatment
}

\author{
Yu-xiao Liư ${ }^{1,2}$, Wen Yue ${ }^{1}$, Lei Ji ${ }^{1}$, Xue Nan ${ }^{1}$, Xue-tao Pei ${ }^{1^{*}}$
}

\begin{abstract}
Background: We recently developed a new method to induce human stem cells (hESCs) differentiation into hematopoietic progenitors by cell extract treatment. Here, we report an efficient strategy to generate erythroid progenitors from hESCs using cell extract from human fetal liver tissue (hFLT) with cytokines. Human embryoid bodies (hEBs) obtained of human H1 hESCs were treated with cell extract from hFLT and co-cultured with human fetal liver stromal cells (hFLSCs) feeder to induce hematopoietic cells. After the 11 days of treatment, hEBs were isolated and transplanted into liquid medium with hematopoietic cytokines for erythroid differentiation. Characteristics of the erythroid cells were analyzed by flow cytometry, Wright-Giemsa staining, real-time RT-PCR and related functional assays.

Results: The erythroid cells produced from hEBs could differentiate into enucleated cells and expressed globins in a time-dependent manner. They expressed not only embryonic globins but also the adult-globin with the maturation of the erythroid cells. In addition, our data showed that the hEBs-derived erythroid cells were able to act as oxygen carriers, indicating that hESCs could generate functional mature erythroid cells.

Conclusion: Cell extract exposure with the addition of cytokines resulted in robust erythroid -like differentiation of hEBs and these hEBs-derived erythroid cells possessed functions similar to mature red blood cells.
\end{abstract}

\section{Background}

Red blood cells (RBCs) have been utilized as the treatment for severe blood loss and hematopoiesis study; but their clinic application has been constrained by limited quantities and compatibility issues. The availability of hESCs offers a great opportunity to produce large quantities of erythroid cells in vitro for transfusion, and to provide additional knowledge to the field of erythropoiesis. Previous studies have generated primitive erythroid cells from hESCs by embryoid body formation and stromal cell co-culturing [1-7]. However, the risk of mouserelated diseases and the low differentiation efficiency of hESCs are major limitations of the clinical application of this study.

Recently, we have established a method to produce relatively large number of human hematopoietic cells from

\footnotetext{
* Correspondence: peixt@nic.bmi.ac.cn

'Stem Cell and Regenerative Medicine Lab, Beijing Institution of Transfusion Medicine, Beijing 100850, China

Full list of author information is available at the end of the article
}

hESCs, via a human-derived induction system, by using hFLSCs feeder cells and cell extract of hFLT. Use of this culture method enabled the production of $32.73 \% \mathrm{CD}^{+} 4^{+}$ from treated hEBs after 11 days of culture. More importantly, hEBs-induced hematopoietic cells predominantly yielded erythroid precursors when seeded on methylcellulose [8]. Based on the above results, we isolated the 11day hEBs from the co-culture system and transplanted them into liquid medium for a 16-day extending culture. During the 16-day culture, cytokines are used to first promote the proliferation and subsequently used for the maturation of erythroid precursors. This culture method enabled the production of about $5 \times 10^{6}$ fully differentiated erythroid cells from about $5 \times 10^{4} \mathrm{hEBs}$. The erythroid cells morphologically resembled fetal liver-derived erythroblasts, they mainly expressed embryonic hemoglobin and could be enucleated. Our results show that induction of hESCs into mature erythroid cells in vitro is possible by treatment with cytokine-supplemented cell extract. 


\section{Results}

The effects of hFLT cell extract treatment on hEBs

After culture on low-attachment plates for about 24 hours, flat hESCs differentiated into typically round hEBs. The permeabilization of hEBs was analyzed with the streptolysin-O (SLO) assay. In a previous study, we found that most hEBs could be labeled with Texas Red containing $700 \mathrm{ng} / \mathrm{ml} \mathrm{SLO} \mathrm{[8],} \mathrm{therefore} \mathrm{we} \mathrm{chose} \mathrm{to}$ incubate hEBs with $700 \mathrm{ng} / \mathrm{ml} \mathrm{SLO}$ for $50 \mathrm{~min}$ in this experiment. After incubation, the permeabilized hEBs were exposed to hFLT cell extract. To reseal cellular plasma membranes, cells were then cultured in IMDM containing $10 \%$ fetal calf serum (FCS) and $2 \mathrm{mM} \mathrm{CaCl}_{2}$. (Figure 1)

\section{The capacity of erythroid-like development of hEBs}

In a previous study, we found that cell extract treatment could influence differentiation of hEBs but only hFLT cell extract treatment could improve hematopoietic differentiation of hEBs [8]. This experiment provided an opportunity to conduct a large-scale investigation of hESCs-derived erythropoiesis after hFLT cell extract treatment. Firstly, we treated hEBs with hFLT cell extract as described previously [8]. Then the treated hEBs were co-cultured on the hFLSCs feeder in hEBs differentiation medium for the hematopoietic differentiation, and the untreated hEBs were culture in the same condition as a control.

To examine the capacity for erythroid development of hEBs, the cells were analyzed by hematopoietic colony assays, and colonies were scored according to their cellular morphology. Our results showed that, for untreated hEBs, the colony-forming cells (CFCs) were first found in the day- $6 \mathrm{hEBs}$ and various types of hematopoietic CFCs increased rapidly afterwards, including colony-forming units-granulocyte-macrophage (CFUGM), colony-forming units- macrophage (CFU-M), colony-forming units-erythroid (CFU-E) colonies, and they reached a peak in day-14 hEBs. As shown in Figure 2, most of the hematopoietic colonies were CFU-GM. At the peak (day 14), about $1 \times 10^{5}$ untreated hEBs-derived cells generated $315.2 \pm 25.3$ hematopoietic colonies, of which about $55 \%$ were CFU-GM and about $22 \%$ were erythroid bursts. For hFLT cell extract treated hEBs, hematopoietic colonies were first detected in day-3 hEBs and they reached a peaked on day 11 . The number of colony-forming cells produced from the treated hEBs was much greater than that from untreated. At the peak, about $1 \times 10^{5}$ untreated hEBs-derived cells could generate $655.2 \pm 31.3$ hematopoietic colonies. Interestingly, hFLT cell extract exposure had a greater effect, both number and frequency, on erythroid colony formation of hEBs. The frequency of CFU-E colonies reached $62 \%$ on day 11 of hEBs (Figure 2). Altogether, these data suggested a supportive role of hFLT cell extract on the erythroid lineage development at the clonal level.

\section{The effects of hFLT cell extract treatment on gene expression}

To understand the effects of hFLT cell extract treatment on gene expression, we investigated the erythroid associated genes of hEBs at different days of co-cultures including SCL, Gata-2, Gata-1, EKLF by RT-PCR. We found that the exposure to hFLT cell extract could promote the erythroid associated gene expression (Figure 3). After incubation with hFLT cell extract, $S C L$ was first detected as early as day 2 and Gata-1 was expressed by day 4, this data confirmed our previous report [8]. The EKLF mRNA was detectable by day 4 of differentiation, and its expression was persistently high throughout the remaining of time course. Gata-2 was obviously expressed by day 8 and up-regulated from day 10 . However, for untreated hEBs, $S C L$ could be first detected by day 4 and Gata- 1 could be found by day 8 at a low level. The other erythroid-associated genes that were tested were difficult to detect before day 12 in the untreated group.

\section{Production of red blood cells from hEBs}

Our data confirmed that hEBs treated with hFLT cell extract possessed a good capacity for erythroid lineage development, and the 11-day hEBs could yield the greatest number of erythroid colonies. To generate a large-scale of

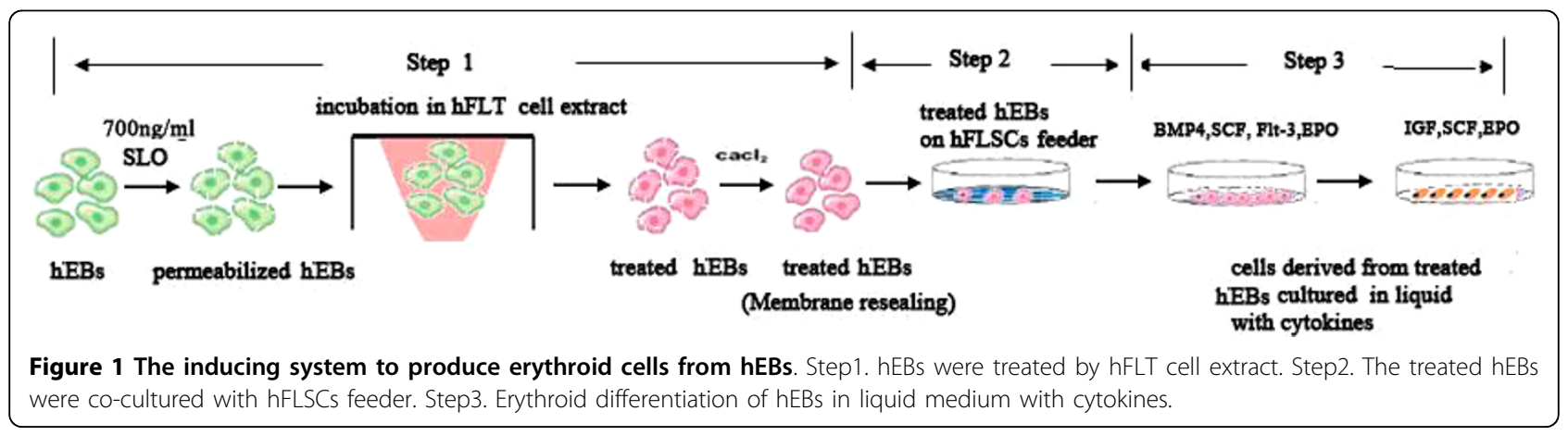




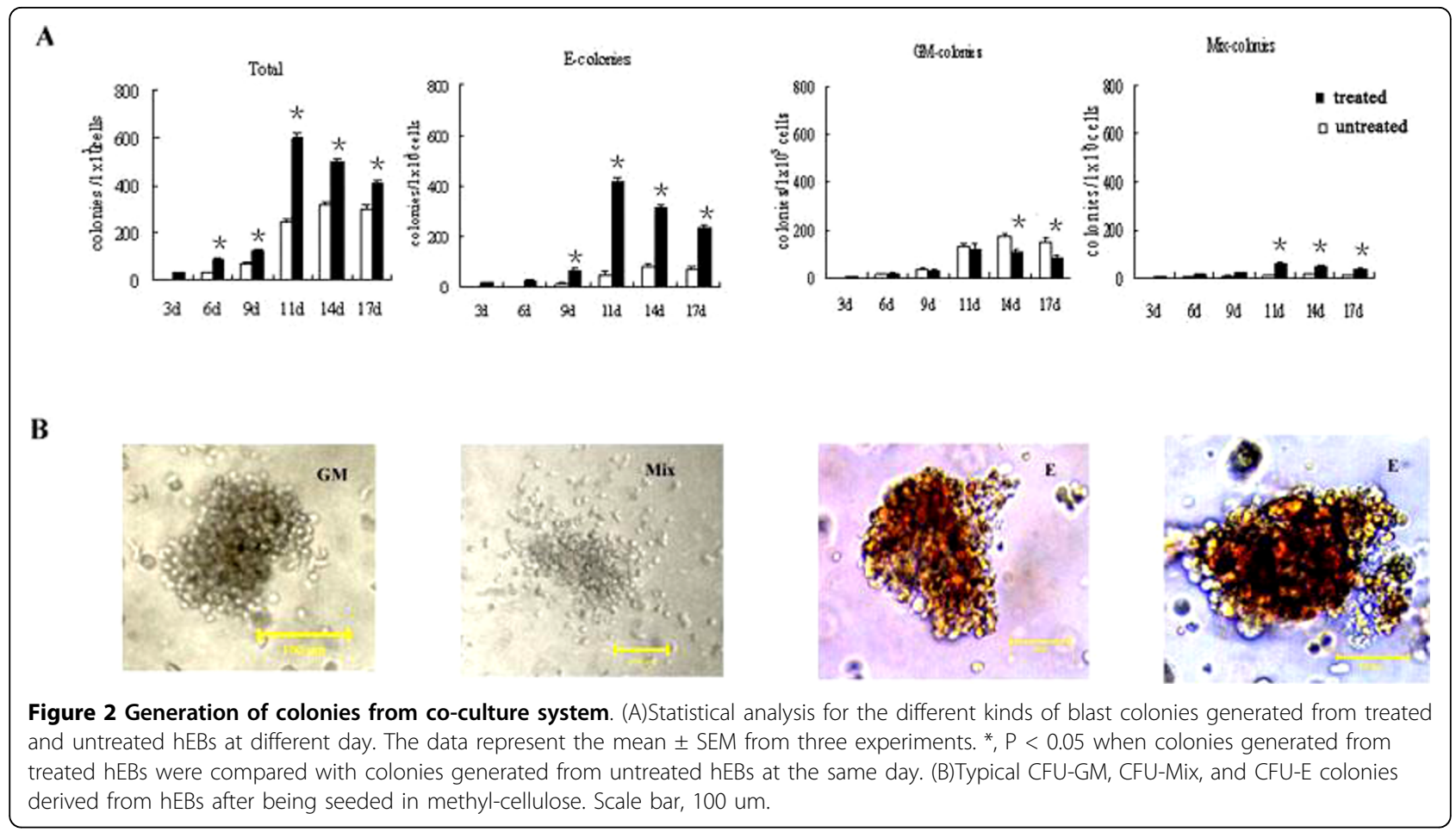

erythroid cells from hESCs, we isolated the 11-day treated hEBs and plated cells in liquid medium. Since bone morphogenetic protein-4 (BMP-4) and Flt-3 ligand (Flt-3l) could promote expanding capacity of hematopoietic cells, so cells were incubated with BMP4 $(20 \mathrm{ng} / \mathrm{mL})$, stem cell factor (SCF, $100 \mathrm{ng} / \mathrm{mL}$ ), erythropoietin (EPO, $2 \mathrm{U} / \mathrm{mL}$ ) and Flt-3l $(20 \mathrm{ng} / \mathrm{mL})$ from day 1 to day 8 . Insulin like growth factor-1 (IGF-1) was necessary for normal erythroid differentiation $[9,10]$, so erythroid progenitors cells were stimulated by incubation with IGF-1 $(20 \mathrm{ng} / \mathrm{mL})$, SCF $(100 \mathrm{ng} / \mathrm{mL})$, EPO (4 U/mL)from day 9 to day 16 . The number of cells was counted during the 16-day liquid culture period. The results showed that the cells expanded rapidly from day 1 to day 8 and the number of cells increased from 50,000 to 5000,000 per well of 24-well plate. However, the cell numbers did not increase from day 9 to day 16 . So about 50,000 hEB cells could yield about 5000,000 erythroid cells during a 16-day incubation, which implied a 100-fold amplification of the erythroid cells produced from the EB cells. (Figure 4A).

To monitor the differentiation of hEB cells into erythroid cells, CD71 (transferrin receptor) and glycophorin A (GLA) antigens were examined by flow cytometry assay throughout the culture. At the beginning of the liquid culture, $\mathrm{CD} 71^{+}$cells were detected at a low lever and $\mathrm{GLA}^{+}$cells could not be detected at all (data not

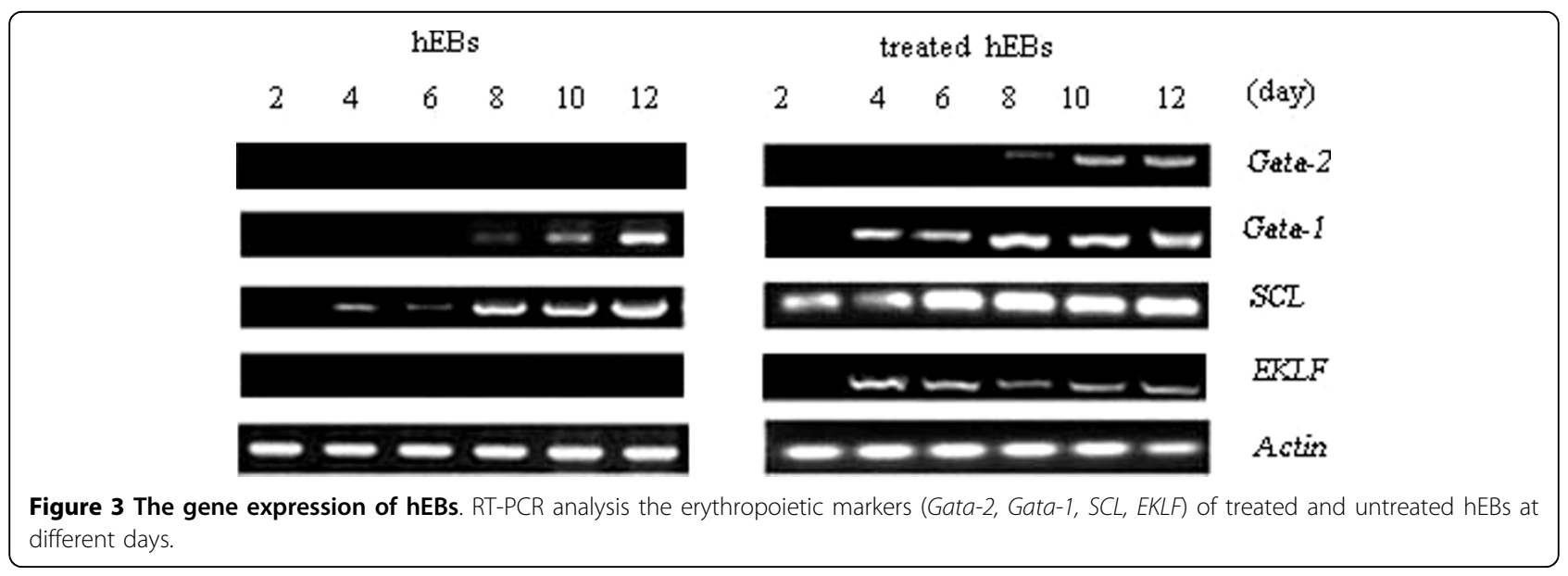



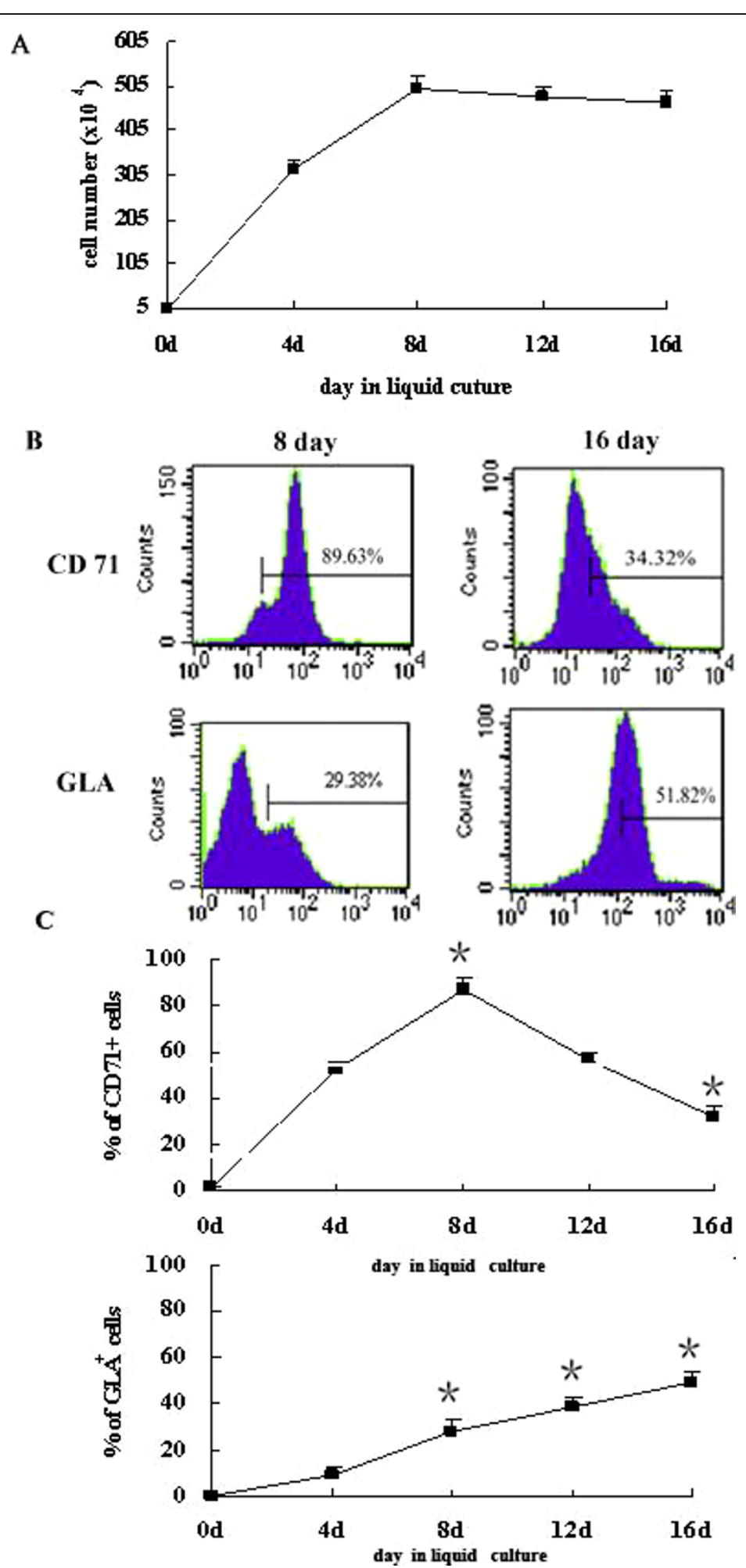

Figure 4 The production of erythroid cells from treated hEBs. (A)Amplification of erythroid cells in liquid culture.50000 hEB cells from coculture could yield about 5000,000 erythroid cells in liquid medium.(B) Analysis of erythroid differentiation of treated hEBs by flow cytometry. Erythroid cells derived from treated hEBs were seeded in liquid medium and tested for expression of CD71 (the marker of early erythroid), GLA (the marker of matuered erythroid) cells by flow cytometry at different inducing times. (C)Statistical analysis for the cell surface antigen expression of hEBs-derived erythroid cells in liquid medium. The data represent the mean \pm SEM from three experiments. ${ }^{*}, \mathrm{P}<0.05$ when compared with the average expression of CD71 or GLA at day 4. 
shown). $\mathrm{CD} 71^{+}$cells increased rapidly from day 1 to day 8 , and reached $89.63 \%$ of their peak level at day 8 and subsequently decreased. About $34 \%$ of the cells were $\mathrm{CD} 1^{+}$cells at the day 16 . In contrast, however, $\mathrm{GLA}^{+}$ cells expanded significantly from day 8 to day 16 . GLA ${ }^{+}$ cells could not reach $30 \%$ at the day 8 , but peaked $51.82 \%$ at the end of culture (Figure 4B, C)

\section{Analysis of hEBs-derived erythroid cells}

To further characterize the erythroid cells derived from hEBs, we stained cells with Wright-Giemsa reagents and compared them with RBCs derived from human fetal liver. We found that hFLT cell extract treatment plus cytokines promoted enucleation of hEBs-derived erythroid cells. As shown in figure 5, the enucleated hEBs-derived RBCs were first detected at day 8 in liquid medium and approximately $20 \%$ to $35 \%$ of erythroid cells were enucleated at day 16 .

We next examined globin expression patterns of these erythroid cells generated from hEBs after 4, 8, 12 or 16 days of culture by real-time PCR(Figure 6A). Then we found that the cells derived from treated hEBs expressed embryonic globins $(\zeta, \varepsilon, \alpha$ and $\gamma)$ but not adult globins $(\beta)$ at the beginning of culture. The expression of $\zeta$ and $\varepsilon$-globins mRNA increased rapidly from day 1 to day 8 , subsequently peaked at day 8 and then decreased. The expression of $\alpha$ and $\gamma$-globins mRNA peaked at day 12 . In contrast, the expression of $\beta$-globin was first detected at day 16 at a low level.

To further observe the expression pattern of globins, we analyzed hEBs-derived erythroid cells at different days by western blotting. We found $\zeta, \varepsilon, \alpha$ and $\gamma$ globins proteins were detected by day 4 and $\beta$-globin protein could be found at day 16 (Figure 6B), which confirmed that erythroid cells generated from hEBs possessed the capacity to express the adult globin at the end of culture. These data indicated that globins expression of erythroid cells generated from hEBs might begin to switch from the embryonic to the adult type at day 16 .

\section{Functional analysis of hEBs-derived erythroid cells}

To assess the function of the hEBs-derived erythroid cells, we measured oxygen dissociation of hEBs-derived erythroid cells and compared them with human cord blood (hCB) and human adult peripheral blood. As shown in Figure 6C, hEBs -derived erythroid cells displayed an oxygen dissociation curve similar to that of $\mathrm{hCB}$, but shifted to the left as compared to the curve of human adult peripheral blood.

\section{Discussion}

In this study, we first established a system to induce the production of a large number of erythroid cells from hESCs utilizing four standard procedures. In the first and second steps, the hEBs were treated with the cell extract from 14-weeks fetal liver and co-cultured with hFLSCs feeders. Cell extract treatment is a novel trans-differentiation strategy that can convert a somatic cell type into another type [11-15]. We recently reported a new method to promote the differentiation of human stem cells toward hematopoietic lineages by the treatment with cell extract of hFLT [8]. The fetal liver is a very important organ of human hematopoiesis, and can generate not only transplantable hematopoietic stem cells, but also enucleated RBCs. We therefore speculated that hFLT cell extract treatment may be advantageous for erythroid line development as well. In the current study, we isolated hEBs from co-cultures after treatment with hFLT cell extract and examined their capacity for the erythroid development at different period of time. At same time the untreated $\mathrm{hEBs}$ were isolated from co-cultures as a control. We found that the treated hEBs in the co-culture system could express the erythroid associated genes and give rise to erythroid lineage colonies in semisolid medium, whereas the untreated hEBs did not express erythroid associated genes and mainly yielded CFU-GM with the same experimental conditions. Thus, our data provided strong evidence which shows
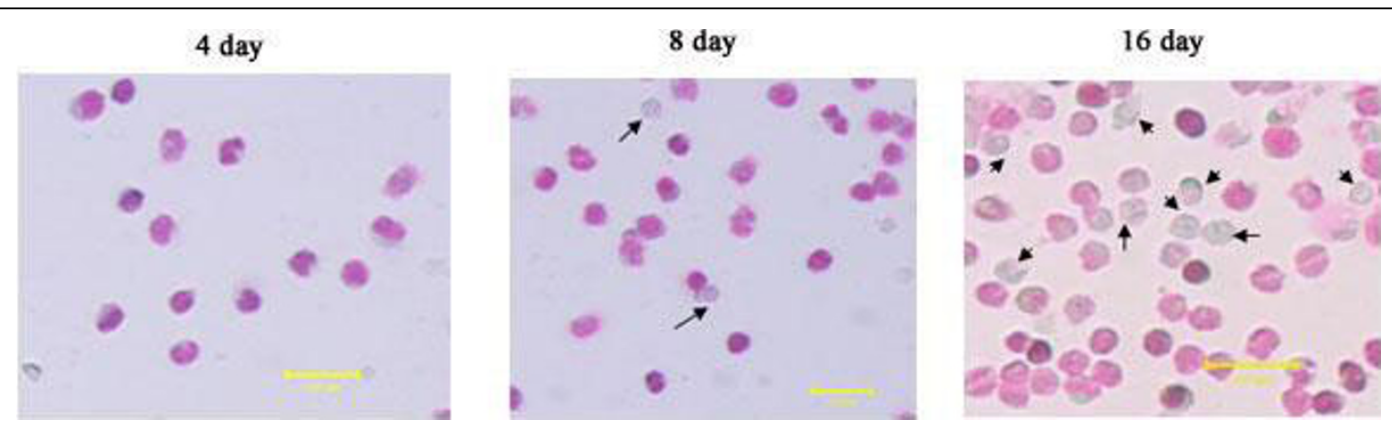

Figure 5 RBCs obtained in liquid medium. The erythroid cells derived from hEBs were stained by Wright-Giemsa reagents at different day (the arrows directed enucleated cells). Scale bar, 100 um. 


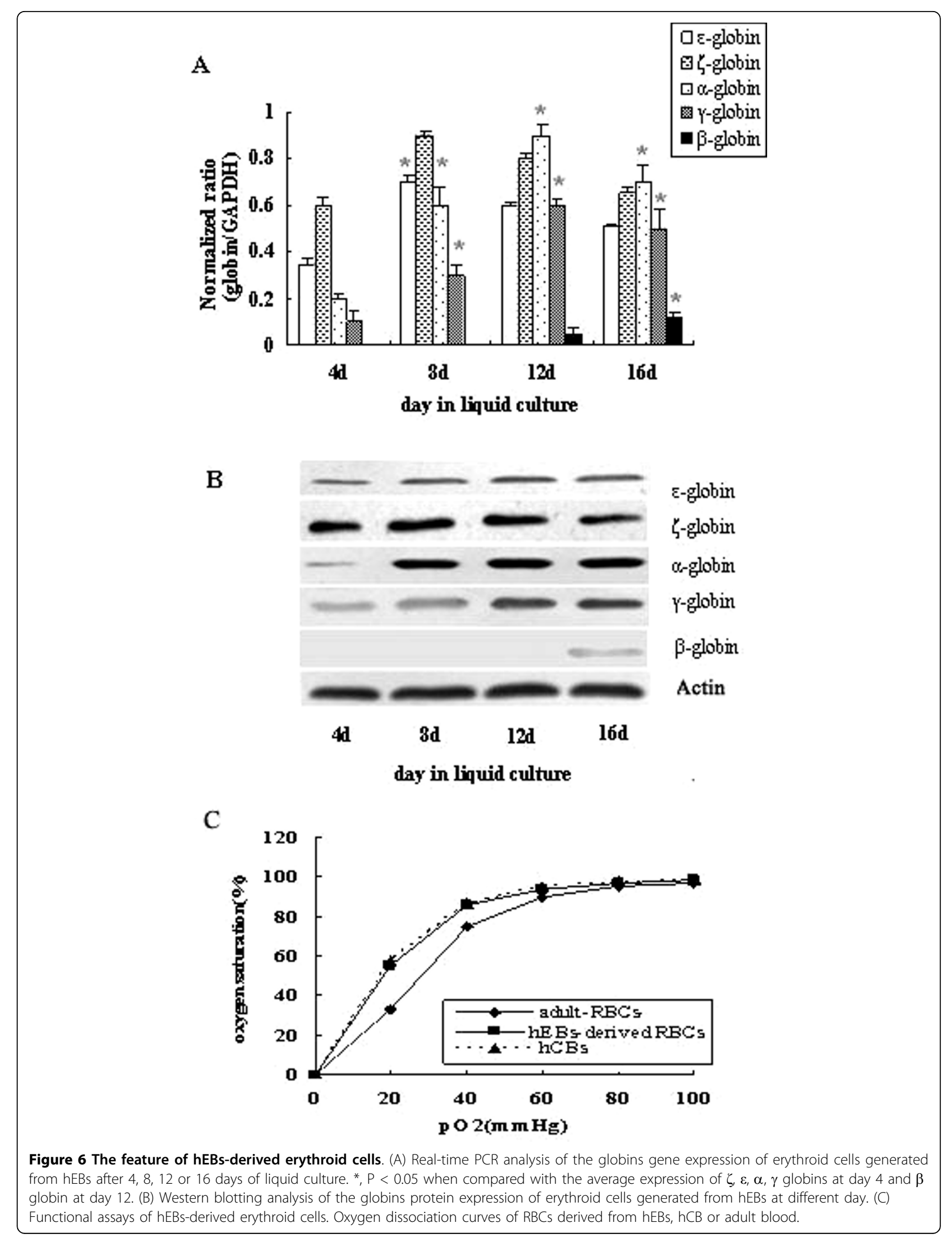


that treated hEBs-derived hematopoiesis mainly generated erythroid cells.

Furthermore, we isolated the treated hEBs from cocultures and induced them into erythroid cells in two -phases. At the beginning of the culture, addition of BMP4, Flt-3l, SCF, and EPO resulted in a rapid increase in cell numbers and an accumulation of differentiated cells. CD71 was used as a marker for early erythroid cells and GLA used as a marker for mature erythroid cells. During the first stage of liquid culture, we observed an obvious increase of $\mathrm{CD} 71^{+}$cells and a slight increase of $\mathrm{GLA}^{+}$cells, which implied the generation and expansion of early erythroid cells from hEBs. From day 9 to day 16, stimulated by IGF-1, SCF and a high dose of EPO, most of the cells had characteristics of mature erythroid cells. During this stage we observed a peak in the percentage of $\mathrm{GLA}^{+}$cells, but the percentage of $\mathrm{CD}_{71}{ }^{+}$cells decreased at the end of culture.

To further understand the erythropoiesis of hESCs, we examined globins expression of erythroid cells from hEBs. According to our previous study, erythroid cells derived from hEBs only expressed embryonic globins but not adult globin [5]. However, in this study we found that hEBs-derived erythroid cells expressed both the embryonic and adult globins at the end of culture. One possible explanation for this result relates to the complex process of mammalian erythropoiesis. In the primitive hematopoiesis wave, blood islands in the yolk sac transiently generate nucleated RBCs. In the later stage of development, the fetal liver is the primary site for production of transplantable hematopoietic stem cells and enucleated RBCs. Definitive hematopoiesis ultimately shifts to the bone marrow, the site for production of life-long adult-type hematopoiesis[2]. Erythrocytes from different hematopoietic sites expressed different types of globins. Yolk sac-derived erythrocytes express only embryonic globins, and erythroblasts produced in fetal liver express embryonic globins and a small amount of adult globin. Erythroblasts from bone marrow primarily expressed adult globin. In our inducing system, hEBs were treated with hFLT cell extract and co-cultured with hFLSCs. It is possible that this condition mimics the environment of the fetal liver and results in similar globin expression of the hEBs-derived erythroid cells as with fetal liverderived erythroid cells.

A critical issue for clinical application of hESCs is whether they can generate functionally mature progenies. In our system, enucleated RBCs could first be observed at day 8 of liquid culture. The number of enucleated RBCs increased rapidly from day 12 to 16 . The erythroid cells produced from treated hEBs morphologically resembled RBCs from human fetal liver. Capability of carrying oxygen is an important function of RBCs. To determine if hEBs-derived erythroid cells possessed the same function as normal RBCs, we analyzed the function of hEBs-derived erythroid cells and found that the oxygen dissociation curve of hEBs-derived erythroid cells shifted to left compared to the curve of human adult blood. To our knowledge, the oxygen dissociation curve of hCB was shifted left when compared to that of human adult peripheral blood [16]. Our results implied that hEBs-derived erythoid cells were also able to function as oxygen carriers and the oxygen dissociation pattern of these cells was more similar to fetal blood cells than adult blood cells. Thus RBCs derived from hEBs in our system may act as an alternative resource of RBCs from blood.

\section{Conclusion}

Hematopoietic differentiation of hESCs has been achieved by using a variety of experimental approaches[1,2]. In addition, the successful derivation of $\mathrm{RBCs}$ from $\mathrm{hCB}$ has been achieved by many published assays[3,4]. However, there are only a few published reports on erythroid differentiation from hESCs. Trans-differentiation of a somatic cell type into another cell type would be beneficial for producing replacement cells for potential therapeutic applications. In this study, we first reported that fetal liver cell extract-based treatment induced the differentiation of hESCs into RBCs safely and efficiently. Our method provides a useful tool for studying the molecular mechanisms of hematopoietic development, and will be valuable in the production of RBCs for transfusion.

\section{Methods}

\section{Cell culture}

hESC H1 cells were obtained from WiCell Research Institute, Inc. (Madison, WI, USA). To prevent cells from differentiating, cells were co-cultured with irradiated (20 cGy) mouse embryonic fibroblast (MEF) cells and maintained in knock-out Dulbecco's modified Eagle's medium (DMEM) supplemented with 20\% serum replacer (SR), $1 \%$ nonessential amino acids (NEAA), $1 \mathrm{mM}$ L-glutamine (all from Invitrogen Corporation, Carlsbad, CA, USA), $0.1 \mathrm{mM} \beta$-mercaptoethanol (Sigma-Aldrich, USA) and $4 \mathrm{ng} / \mathrm{ml}$ human basic fibroblast growth factor (bFGF) (R\&D Systems, USA).

The hFLSCs were isolated and cultured as previously described [17].

\section{Preparation of cell extract}

The hFLT cell extract was prepared, as described previously $[18,19]$, from 15 -week human aborted fetal liver tissue obtained with informed consent. Briefly, the hFLT cell extract was prepared as follows: cells were washed in phosphate-buffered saline (PBS) and resuspended in cell lysis buffer. Cells were lysed with a dropping pipette and centrifuged at $15,000 \times g$ for 15 minutes at $4^{\circ} \mathrm{C}$. The supernatant was removed, filtered and stored at $-80^{\circ} \mathrm{C}$. 
Table 1 Primer sequences for RT-PCR

\begin{tabular}{lll}
\hline Name & Primer sequence (sense) & Primer sequence (antisense) \\
\hline Gata-1 & CAGGTACTGCCCATCTCTAC & TCTGGCTACAA G AGGAGAAG \\
Gata-2 & AAGGCTCGTTCCTGTTCAGA & GGCATTGCACAGGTAGTGG \\
SCL & GCTGGCTITCTGTTCCTG & TGACA ACCCCAGGTCTTA GG \\
EKLF & CGGACACACAGGATGATCTC & GGCTGGTCCTCAGACTTCAC \\
$\beta$-Actin & GATCCACATCTGCTGGAAGG & AAGTGTGACGTTGACATCCG \\
\hline
\end{tabular}

\section{Inducing erythroid differentiation of hEBs}

\section{Step 1. Cell Extract treatment of hEBs}

Undifferentiated hESCs were harvested at $80 \%$ confluence after collagenase IV digestion and transferred to $10-\mathrm{cm}$ low cell-binding dishes to form hEBs in hEBs medium containing KO-DMEM supplemented with 20\% FBS, 1\% NEAA, 1 $\mathrm{mM}$ L-glutamine, and $0.1 \mathrm{mM} \beta$-mercaptoethanol. The hEBs were then treated with hFLT extract. Briefly, the 2day hEBs were treated with SLO in $\mathrm{Ca}^{2+-} \mathrm{Mg}^{2+}$-free Hanks' balanced salt solution (Gibco-BRL, Invitrogen) for $50 \mathrm{~min}$ utes at $37^{\circ} \mathrm{C}$. About $100 \mu \mathrm{l}$ of hFLT cell extract containing an XX ul or \% ATP-generating system (1 mM ATP, $1 \mathrm{mM}$ GTP, $1 \mathrm{mM}$ NTP, $10 \mathrm{mM}$ phosphocreatine, and $25 \mu \mathrm{g} / \mathrm{ml}$ creatine kinase, Sigma) were added to replace the SLO and incubated for 60 minutes at $37^{\circ} \mathrm{C}$. To reseal plasma membranes $\mathrm{CaCl}_{2}(2 \mathrm{mM})$ was added to the hESCs culture medium, and cells were cultured overnight at $37^{\circ} \mathrm{C}$.

\section{Step 2. hFLSCs feeder co-culture}

The treated hEBs were cultured with hFLSCs feeder in hEBs differentiation medium for 11 days containing $80 \%$ IMDM (Invitrogen), 30\% FBS (Invitrogen), 1\% NEAA, 2 $\mathrm{mM}$ L-glutamine, $0.1 \mathrm{mM}$ beta-mercaptoethanol. After some days of culture, cells were collected and analyzed to identify erythroid differentiation. Total RNA was isolated from hEBs at different days of co-culture using the following the manufacturer's protocol. We analyzed mRNA expression for markers of erythroid cells by RT-PCR. In addition, the EB cells at different days of co-culture were collected and seeded in MethoCult GF -H4434 semisolid medium (Stem Cell Technologies, USA) to determine their capacity for erythroid development.

\section{Step 3. Erythroid differentiation of hEBs in liquid medium with cytokines}

The hEBs that possessed the greatest capacity for erythroid development were dissociated into single-cell suspensions by collagenase IV treatment. Cells were induced into erythroid cells by two phases: from day 1 to day 8 , cells were cultured in medium composed of IMDM supplemented with $30 \%$ FBS, BMP4 (20 ng/mL), SCF (100 ng/mL), EPO (2 U/mL), and Flt-3l(20 ng/mL); from day 9 to day 16, cells were cultured in medium composed of IMDM supplemented with $30 \%$ FBS, IGF-1 (20 ng/mL), SCF (100 ng/mL), EPO (4 U/mL). About 1 to $2 \mathrm{~mL}$ fresh medium was added to each plate every 2 to 3 days,.

\section{RT-PCR}

The gene expression of hEBs was examined by RT-PCR. All PCR reactions were performed as follows: $95^{\circ} \mathrm{C}$ for $5 \mathrm{~min}$; $94^{\circ} \mathrm{C}$ for $40 \mathrm{~s}$; annealing at various temperatures for $40 \mathrm{~s}$, $72^{\circ} \mathrm{C}$ for $40 \mathrm{~s}$ ( 25 cycles); $72^{\circ} \mathrm{C}$ for $10 \mathrm{~min}, 4^{\circ} \mathrm{C}$ for $5 \mathrm{~min}$. The forward and reverse primers are shown in Table 1 :

\section{Clonogenic progenitor cell assay}

Hematopoietic clonogenic assays were performed in 35$\mathrm{mm}$ low adherent plastic dishes using MethoCult GF -H4434 semisolid medium (Stem Cell Technologies, USA) consisting of $1 \%$ methylcellulose, 30\% FBS, $1 \%$ bovine serum albumin (BSA), $50 \mathrm{ng} / \mathrm{mL}$ stem cell factor, $20 \mathrm{ng} / \mathrm{mL}$ granulocyte-macrophage colony-stimulating factor (GM-CSF), $20 \mathrm{ng} / \mathrm{mL}$ IL-3, $20 \mathrm{ng} / \mathrm{mL}$ IL-6, $20 \mathrm{ng} /$ $\mathrm{mL}$ granulocytecolony-stimulating factor (G-SF), and 3 units/mL erythropoietin(EPO). After culturing for 12-14 days, colonies were scored according to their cellular morphology.

\section{Real-Time PCR}

The expression of globins in erythriod cells was examined by real-time RT-PCR using the SYBR Green PCR Master Mix. All PCR reactions were performed as follows: $95^{\circ} \mathrm{C}$ for $5 \mathrm{~min}$; $94^{\circ} \mathrm{C}$ for $40 \mathrm{~s}$; annealing at various temperatures for $40 \mathrm{~s}, 72^{\circ} \mathrm{C}$ for $40 \mathrm{~s}$ ( 25 cycles); $72^{\circ} \mathrm{C}$ for $10 \mathrm{~min}, 4^{\circ} \mathrm{C}$ for $5 \mathrm{~min}$. The forward and reverse primers used were described in Table 2 as follows:

\section{Western blotting}

The cell extract was subjected to sodium dodecyl sulfate-polyacrylamide gel electrophoresis (SDS-PAGE) and transferred onto a polyvinylidene difluoride membrane

Table 2 Primer sequences for Real-time PCR

\begin{tabular}{lll}
\hline Name & Primer sequence $($ sense) & Primer sequence (anti-sense) \\
\hline $\boldsymbol{\zeta}$-globin & GCTCAGGCCGAGCCCATTGG & TAGCGGTACTTCTCAGTCAG \\
$\boldsymbol{\varepsilon}$-globin & GGAGAGTCCATTAAGAATCTA & CTGTGAATTCATTGCCGA AGT \\
$\beta$-globin & CGGTCAACTTCAAGCTCCTAAG & CCGCCCACTCAGACTITATT \\
$\gamma$-globin & TACATTGCTTCTGACACAAC & ACAGATCCCCAAAGAC \\
GAPDH & CTCAAGCTCCTGGGAATGT & GCAGAATAAAGCCTACCTTGAAAG \\
\hline
\end{tabular}


(Millipore, Bedford, MA). After blocking in TBST containing 5\% dry-fat skim milk, the membrane was incubated with anti-globin antibodies (Santa Cruz Biotechnology), followed by HRP-labeled goat anti rabbit IgG. The protein bands were visualized by enhanced chemiluminescence (ECL; Pierce).

\section{Flow cytometric analysis}

The trypsinized individual cells were incubated with the following FITC-conjugated and PE-conjugated monoclonal antibodies: anti-human CD71, anti-human glycophorin A (B\&D Biosciences, USA) for 30 minutes at $4^{\circ}$ C. Cells were washed with PBS for three times, and analyzed by flow cytometeric analysis using the FACSCalibur (Becton-Dickinson, Mountain View, CA, USA).

\section{Wright-Giemsa staining}

Cells were dropped onto slides and fixed for 20 minutes in $4 \%$ paraformaldehyde and were stained with WrightGiemsa reagents (Fisher Scientific) following manufacturer's instructions.

\section{Functional Assays for Erythroid Cells}

The oxygen binding ability of hESCs-derived erythroid cells and human adult blood was measured with a Hemox analyzer, as reported previously [20,21].

\section{Statistical analysis}

Results were expressed as means \pm SEM. Statistical significance was determined using Student $\mathrm{t}$ - test. Results were considered significant at $\mathrm{P}<.05$.

\section{Acknowledgements}

This work was supported by National High Technology Research and Development Program of China (No:2006AA02A107), the Major State Basic Research Program of China (No:2005CB522702), and The Project of Beijing Municipal Science \& Technology Commission (No: Z0005190043331)

\section{Author details}

${ }^{1}$ Stem Cell and Regenerative Medicine Lab, Beijing Institution of Transfusion Medicine, Beijing 100850, China. ${ }^{2}$ Department of Neurosurgery, the First Affiliated Hospital of PLA General Hospital, Beijing 100037, China.

\section{Authors' contributions}

$Y X L$ participated in the design of the study and carried out most of the experiments. XTP and WY participated in the coordination and design of the study. WY also helped in drafting the manuscript. $L$ helped in cell culture and helped draft the manuscript. XN helped with RT-PCR. All authors read and approved the final manuscript.

Received: 1 January 2010 Accepted: 10 August 2010 Published: 10 August 2010

\section{References}

1. Kaufman DS, Hanson ET, Lewis RL, Auerbach R, Thomson JA: Hematopoietic colony-forming cells derived from human embryonic stem cells. Proc Natl Acad Sci USA 2001, 98:10716-10721.

2. Qiu C, Hanson E, Olivier E, Inada M, Kaufman DS, Gupta S, Bouhassira EE: Differentiation of human embryonic stem cells into hematopoietic cells by co-culture with human fetal liver cells recapitulates the globin switch that occurs early in development. Experimental Hematology 2005, 33:1450-1458.

3. Chang $\mathrm{KH}$, Nelson $\mathrm{AM}$, $\mathrm{CaO} \mathrm{H}$, Wang $L$, Nakamoto $B$, Ware $C B$, Papayannopoulou T: Definitive- like erythroid cells derived from human embryonic stem cells coexpress high levels of embryonic and fetal globin swith little or no adult globin. Blood 2006, 108:1515-1523.

4. Cerdan Chantal, Rouleau Anne, Bhatia Mickie: VEGF-A165 augments erythropoietic development from human embryonic stem cells. Blood 2004, 103:2504-2512

5. Olivier NEmmanuel, Qiu Caihong, Velho Michelle, Hirsch Elison Rhoda, Eric E: Large-scale production of embryonic red blood cells from human embryonic stem cells. Experimental Hematology 2006, 34:1635-1642

6. Otani Takeshi, Inoue Toshiya, Tsuji-Takayama Kazue, ljiri Yoshihiro, Nakamura Shuji, Motoda Ryuichi, Orita Kunzo: Progenitor analysis of primitive erythropoiesisgenerated from in vitro culture of embryonic stem cells. Experimental Hematology 2005, 33:632-640.

7. Ma F, Ebihara Y, Umeda K, Sakai H, Hanada S, Zhang H, Zaike Y, Tsuchida E, Nakahata T, Nakauchi H, Tsuji K: Generation of functional erythrocytes from human embryonic stem cell-derived definitive hematopoiesis. PNAS 2008, 05(35):13087-92.

8. Liu Yu-xiao, Ji Lei, Yue Wen, Yan Zhi-feng, Wang Jing, Xi Jia-fei, Zhang Rui, Nan Xue, Bai Ci-xian, Chen Lin, Wang Yun-fang, Pei Xue-tao: Cells Extract from Fetal Liver Promotes the Hematopoietic Differentiation of Human Embryonic Stem Cells. Cloning Stem Cells 2009, 11:51-60.

9. Sawada K, Krantz SB, Dessypris EN, Koury ST, Sawyer ST: Human colonyforming units-erythroid do not require accessory cells, but do require direct interaction with insulin-like growth factor I and/or nsulin for erythroid development. J Clin Invest 1989, 83:1701-1709.

10. Muta K, Krantz SB, Bondurant MC, Wickrema A: Distinct roles of erythropoietin, insulin-like growth factor I, and stem cell factor in the development of erythroid progenitor cells. J Clin Invest 1994, 94:34-43.

11. Gaustad KG, Boquest AC, Anderson BE, Gerdes AM, Collas P: Differentiation of human adipose tissue stem cells using extract of rat cardiomyocytes. Biochem Biophys Res Commun 2004, 314:420-427.

12. Håkelien AM, Landsverk HB, Robl JM, Skålhegg BS, Collas P: Reprogramming fibroblasts to express T-cell functions using cell extract. Nat Biotechnol 2002, 20:460-466.

13. Hakelien AM, Gaustad KG, Collas P: Transient alteration of cell fate using a nuclear and cytoplasmic extract of an insulinoma cell line. Biochem Biophys Res Commun 2004, 316:834-841.

14. Jaenisch R, Young R: Stem Cells, the Molecular Circuitry of Pluripotency and Nuclear Reprogramming. Cell 2008, 132:567-582.

15. Gurdon JB, Melton DA: Nuclear Reprogramming in Cells. Science 2008, 322:1811-15.

16. Ma F, Ebihara Y, Umeda K, Sakai H, Hanada S, Zhang H, Zaike Y, Tsuchida E, Nakahata T, Nakauchi H, Tsuji K: Generation of functional erythrocytes from human embryonic stem cell-derived definitive hematopoiesis. PNAS 2008, 105:13087-92.

17. Ji L, Liu YX, Yang C, Yue W, Shi SS, Bai CX, Xi JF, Nan X, Pei XT: Self-renewal and pluripotency is maintained in human embryonic stem cells by coculture with human fetal liver stromal cells expressing hypoxia inducible factor 1alpha. J Cell Physiol 2009, 221(1):54-66.

18. Collas $P$, Taranger CK: Epigenetic reprogramming of nuclei using cell extracts. Stem Cell Rev 2006, 2:309-317.

19. Qin M, Tai G, Collas P, Polak JM, Bishop AE: Cell Extract-Derived Differentiation of Embryonic Stem Cells. Stem Cells 2005, 23:712-718.

20. Honig GR, VidaL N, Latorraca R, Divgi AB: Hb south Milwaukee [beta1 05 (G7) Leu-Phe ]:a newly-identified hemoglobin variant with high Oxygen affinity. Am J Hematol 1990, 34:199-203.

21. Sakai $H$, Cabrales $P$, Tsai $A-G$, Intaglietta $M$, Tsuchida E: Oxygen release from low and normal P50 Hb-vesicles in transiently occluded arterioles of the hamster window model. Am J Physiol Heart Circ Physiol 2005, 288(6): $\mathrm{H} 2897-\mathrm{H} 2903$.

doi:10.1186/1471-213X-10-85

Cite this article as: Liu et al:: Production of erythriod cells from human embryonic stem cells by fetal liver cell extract treatment. $B M C$

Developmental Biology 2010 10:85 